\title{
Early skin-to-skin contact or incubator for very preterm infants: study protocol for a randomized controlled trial
}

Laila Kristoffersen ${ }^{1,2^{*}}$, Ragnhild Støen ${ }^{1,2}$, Hilde Rygh ${ }^{1}$, Margunn Sognnæs ${ }^{3}$, Turid Follestad ${ }^{4}$, Hilde S. Mohn ${ }^{5,6}$, Ingrid Nissen ${ }^{1,6}$ and Håkon Bergseng ${ }^{1,2}$

\begin{abstract}
Background: Skin-to-skin care immediately following delivery is a common practice for term infants and has been shown to improve cardiorespiratory stability, facilitate early bonding, and promote breastfeeding. Since 2007, the use of skin-to-skin care has been practiced for preterm infants from 32 weeks of gestation in the delivery room at St. Olav's University Hospital. In the present study we aim to investigate whether skin-to-skin care following delivery is safe, and how it affects early and late outcomes compared to standard care for very preterm infants.

Methods/Design: A randomized controlled trial (RCT) of skin-to-skin care in the delivery room for very preterm infants born at gestational age $28^{0}-31^{6}$ weeks with birth weight $>1000$ grams. Infants with severe congenital malformations or need of intubation in the delivery room are excluded. A detailed checklist and a flowchart were prepared for the study, and all involved professionals (neonatologists, neonatal nurses, obstetricians, anesthesiologists, midwives) participated in medical simulation training prior to study start on February 1, 2014. A consultant in neonatology and a neonatal nurse are present at all deliveries. Infants with birth weight $<1500$ grams receive an intravenous line with glucose, amino acids, and caffeine citrate in the delivery room. Infants with gestational age $<30$ weeks are routinely put on continuous positive airway pressure (CPAP). After initial stabilization, infants are randomized to skin-to-skin care or are transferred to the nursery in an incubator. Primary outcome is cognitive development at 2 years measured with the Bayley Scales of Infant Development, Third Edition. Secondary outcomes are safety defined as hypothermia, respiratory failure, and/or cardiopulmonary resuscitation, physiological stability after birth and motor, language and cognitive development at 1 year for the child, and mental health measured with the State-Trait Anxiety Inventory (STAl) at discharge, and at 3 months and 2 years after expected date of delivery for the mothers.
\end{abstract}

Discussion: The study may have important implications for the initial care for very preterm infants after delivery and increase our understanding of how early skin-to-skin care affects preterm infants and their mothers.

Trial registration: ClinicalTrials, NCT02024854. Registered on 19 December 2013.

Keywords: Very preterm infants, Early skin-to-skin, Kangaroo mother care, Neonatal intensive care unit

\footnotetext{
* Correspondence: Laila.kristoffersen@ntnu.no

${ }^{1}$ Department of Pediatrics, St. Olav's University Hospital, Trondheim, Norway

²Department of Laboratory Medicine, Children's and Women's Health,

Norwegian University of Science and Technology, NTNU, Trondheim, Norway

Full list of author information is available at the end of the article
}

(c) The Author(s). 2016 Open Access This article is distributed under the terms of the Creative Commons Attribution 4.0 International License (http://creativecommons.org/licenses/by/4.0/), which permits unrestricted use, distribution, and reproduction in any medium, provided you give appropriate credit to the original author(s) and the source, provide a link to the Creative Commons license, and indicate if changes were made. The Creative Commons Public Domain Dedication waiver (http://creativecommons.org/publicdomain/zero/1.0/) applies to the data made available in this article, unless otherwise stated. 


\section{Background}

Worldwide, 15 million infants are born preterm annually [1], and preterm birth is one of the largest direct causes of neonatal mortality and morbidity [2]. Compared to full-term infants, preterm infants are at increased risk of neurodevelopmental impairments [3-5]. This also includes lower self-esteem, social relations, and quality of life in adulthood [6]. There is growing evidence that mental and behavioral problems in children born preterm last into adulthood [7-13]. These societal and medical consequences of preterm births have led to a growing interest in developmental care models to optimize neurodevelopmental outcomes. Different approaches aiming to support infants and parents have been established in neonatal intensive care units (NICUs) in recent years, and some of these early intervention programs seem to have a positive effect on longterm function up to preschool age $[5,14,15]$. The first hours after birth represent a sensitive period for the very low birthweight infant (VLBW), and mothers who see their infant within 3 hours after birth are likely to establish a more secure attachment to the infant compared to those who do not see their infant within 3 hours [16]. Parents are increasingly acknowledged as primary caregivers for their preterm-born infant; despite the need for intensive care, most NICUs try to facilitate early parentinfant bonding. Education of parents to understand subtle signs and signals from their tiny, preterm-born infants is considered an investment in an optimal home environment for the child. From being forced to separate from their newborn infant due to restrictions of visiting hours in NICUs, parents are now generally encouraged to stay with their infant as much as possible $[17,18]$.

Kangaroo care (KC) [19], family-centered care (FCC) [20], Newborn Individualized Development Care and Assessment Program (NIDCAP) [21, 22], and a variety of early intervention programs emphasize the importance of establishing early parent-infant interaction to support an optimal development [23-25]. Facilitating early skin-to-skin care (SSC) is one way of supporting early parent-infant bonding and is also associated with improved physiological stability [26, 27] and decreased cortisol reactivity [28]. In addition, a recent World Health Organization (WHO) guideline on interventions to improve outcomes for preterm infants, strongly recommends early SSC as thermal care for preterm infants weighing <2000 grams [29].

Skin-to-skin care in the delivery room (DR) has been studied for preterm infants from 32 weeks of gestation [30]. However, to the best of our knowledge, SSC in the DR and the operating room (OR) has not been systematically investigated for infants born at gestational age (GA) $<32$ weeks. Obvious obstacles to do so would be the need for medical equipment and competent personnel, usually available only in the NICU. Breathing support, need of surfactant, intravenous access, and monitoring equipment are needed in a large proportion of infants below 32 weeks. To combine medical interventions with early SSC, a trained team and formalized guidelines are required to ensure the medical safety of the infant. Specific challenges arise with early SSC in the OR after cesarean section (C-section).

Moving treatment and medical procedures from the NICU into the DR and OR requires cooperation across specialties and departments. Pediatricians and neonatal nurses have to work outside their familiar NICU environment, while gynecologists, midwives, anesthesiologists, nurse specialists in anesthesia, and operating room nurses have to adjust their work and procedures to the presence of the preterm infant on the mother's chest.

In the present study we aim to investigate whether SSC following delivery is safe, and how it affects early and late outcomes compared to standard care for preterm infants born at GA $28^{0}-31^{6}$ weeks.

The purpose of this article is to present the study design and a description of the intervention, as well as provide a detailed outline of how the intervention was planned and implemented in a multidisciplinary team. Schedule of enrolment, interventions, and assessment are outlined in Table 1.

\section{Aims \\ Primary aim}

To study the effect of early SSC versus standard care (SC) for very preterm infants $\left(28^{0}-31^{6}\right.$ weeks $)$ on cognitive scores at 2 years corrected age, measured with the Bayley Scales of Infant Development, Third Edition (Bayley-III).

\section{Secondary aims}

- To assess safety of the intervention by measuring incidence of hypothermia (temperature $<36{ }^{\circ} \mathrm{C}$ ) or respiratory failure during the first 2 hours of life.

- To estimate the effect of the intervention on complications to prematurity (intraventricular hemorrhage (IVH) or periventricular leukomalacia (PVL), seizures, necrotizing enterocolitis (NEC), treatment for persistent ductus arteriosus (PDA), and bronchopulmonary dysplasia (BPD)).

- To estimate the effect of the intervention on physiological stability during the first 24 hours.

- To estimate the effect of the intervention on maternal mental health at discharge from the NICU and when the child's corrected age is 3 months and again at the corrected age of 2 years by using the State-Trait Anxiety Inventory (STAI).

- To investigate if the intervention affects the fidgety type of general movements and detailed aspects of 
Table 1 Schedule of enrolment, interventions, and assessment

\begin{tabular}{|c|c|c|c|c|c|c|c|c|c|c|c|c|c|c|c|c|}
\hline Activity/assessment & Staff member & $\begin{array}{l}\text { Approximate time to } \\
\text { complete }\end{array}$ & $\begin{array}{l}\text { Before } \\
\text { delivery }\end{array}$ & $t_{0}$ & $t_{1}$ & $t_{2}$ & $t_{3}$ & $t_{4}$ & $t_{5} t$ & $\begin{array}{ll}t_{6} & t_{7}\end{array}$ & $7 t_{8}$ & $t_{9}$ & $t_{10} t_{1}$ & $t_{12}$ & $t_{13}$ & $\mathrm{t}_{14}$ \\
\hline Informed consent & $\begin{array}{l}\text { Neonatal nurse/ } \\
\text { physician }\end{array}$ & 15 minutes & $x$ & & & & & & & & & & & & & \\
\hline Inclusion/exclusion form & Neonatal nurse & 5 minutes & & $x$ & & & & & & & & & & & & \\
\hline Randomization sealed envelopes & Neonatal nurse & 1 minute & & & $x$ & & & & & & & & & & & \\
\hline Heart rate & Neonatal nurse & $30 \mathrm{sec}$ & & & & $x$ & $x$ & $x>$ & $x \quad x$ & $x$ & & & & & & \\
\hline Respiration rate & Neonatal nurse & $30 \mathrm{sec}$ & & & & $x$ & $x$ & $x>$ & $x \quad x$ & $x$ & & & & & & \\
\hline Oxygen saturation & Neonatal nurse & $30 \mathrm{sec}$ & & & & $x$ & $x$ & $x>$ & $x \quad x$ & $x$ & & & & & & \\
\hline Body temperature & Neonatal nurse & $30 \mathrm{sec}$ & & & & $x$ & $x$ & $x>$ & $x$ & & $x$ & $x$ & & & & \\
\hline Blood glucose & Neonatal nurse & 5 minutes & & & & & & $x^{1}$ & $x^{1}$ & $x$ & & & $x$ & & & \\
\hline Blood pressure & Neonatal nurse & 5 minutes & & & & & & & $x$ & & $x$ & $x$ & & & & \\
\hline Maternal anxiety (STAl) & Study coordinator & 15 minutes & & & & & & & & & & & $x$ & $x$ & & $x$ \\
\hline Ages \& Stages Questionnaire & Study coordinator & 15 minutes & & & & & & & & & & & & $x$ & & $x$ \\
\hline General movement (GMA) & Study coordinator & 5 minutes & & & & & & & & & & & & $x$ & & \\
\hline $\begin{array}{l}\text { Bayley Scale of Infant and Toddler } \\
\text { Development III }\end{array}$ & $\begin{array}{l}\text { Occupational } \\
\text { therapist }\end{array}$ & 60 minutes & & & & & & & & & & & & & $x$ & $x$ \\
\hline
\end{tabular}

$\mathrm{X}^{1}=$ the blood glucose is measured at either 90 or 120 minutes

$\mathrm{t}_{0}=$ During stabilization

$\mathrm{t}_{1}=$ After initial stabilization

$\mathrm{t}_{2}=15$ minutes after birth

$\mathrm{t}_{3}=30$ minutes after birth

$\mathrm{t}_{4}=60$ minutes after birth

$t_{5}=120$ minutes after birth

$\mathrm{t}_{6}=$ First 24 hour (hourly)

$t_{7}=$ Approximately 12 hours after birth

$\mathrm{t}_{8}=10$ hours after birth

$\mathrm{t}_{9}=18$ hours after birth

$\mathrm{t}_{10}=$ Approximately 24 hours after birth

$\mathrm{t}_{11}=$ At discharge

$\mathrm{t}_{12}=3$ months corrected age

$\mathrm{t}_{13}=1$ year corrected age

$\mathrm{t}_{14}=2$ years corrected age

the early motor repertoire at the corrected age of 3 months, using the General Movement Assessment (GMA) and the Assessment of Motor Repertoire - 2 to 5 months (AMR).

- To estimate the effect of the intervention on neurodevelopmental outcomes including cognition, language, and motor function at the corrected age of 1 year, and language and motor function at the corrected age of 2 years assessed with Bayley-III.

- To investigate the effect of the intervention on social and emotional competence at the corrected age of 3 months and at 2 years with the Ages \& Stages Questionnaire - Social-Emotional

\section{Methods/Design}

\section{Project context}

The study is currently being carried out at St. Olav's University Hospital in Trondheim. Trondheim is the third largest city in Norway, and St. Olav's Hospital serves a population of almost 446,000 and is a tertiary care center for preterm infants $<30$ weeks of gestation.
Approximately 50 infants with GA $<32$ weeks are born every year at St. Olav's Hospital.

The NICU at St. Olav's Hospital has 21 beds, with seven beds for intensive care, seven intermediate, and seven in a step-down unit. Altogether, there are 107 nurses (many part-time) and six consultants working in the NICU. The maternity ward has two sections with seven delivery rooms each and about 120 midwives employed.

Delivery rooms are in the same building as the NICU, but on separate floors (fifth and second floor, respectively). The operating room and the postoperative ward are in the same building and the same floor as the NICU.

\section{Study design}

The study is designed as an RCT with randomization to skin-to-skin care (SSC) or standard care (SC) in an incubator after delivery.

\section{Study population}

Singleton and twin preterm infants with GA $28^{0}-31^{6}$ and a BW >1000 grams in a stable medical condition delivered 
either vaginally or by $\mathrm{C}$-section are candidates for inclusion. Twins are randomized to the same intervention. Infants with severe malformations not compatible with life, or requiring surgery within hours to days after birth (e.g. esophageal atresia, abdominal wall defects or neural tube defects), will be excluded.

Infants who need intubation and mechanical ventilation, or CPAP with more than $40 \%$ oxygen to maintain a saturation above $90 \%$ at 10 minutes of age, are excluded. Mothers have to be awake (not under general anesthesia) during $\mathrm{C}$-section.

\section{Intervention}

For all infants of eligible women, cord milking is advised immediately following delivery and before the infant is placed on the resuscitation unit. Predefined blood samples are taken from the umbilical cord. Blood gas is taken from the umbilical artery before cord clamping, while blood for hemoglobin, leukocytes, thrombocytes, C-reactive protein (CRP) and blood typing is sampled after the cord clamping.

After the initial stabilization, the infant is treated and evaluated according to checkpoint 1 on the checklist, and eligibility is decided by the consultant (Fig. 1). Infants randomized to standard care are transferred to the NICU in an incubator after stabilization. The father will usually follow the newborn to the NICU, while the mother will come as soon as possible after delivery.

All infants with a BW $<1500 \mathrm{~g}$, irrespective of randomization, are given parenteral nutrition (glucose and amino acids) after the initial stabilization. Infants with a GA $<30$ weeks receive CPAP, infants $\geq 30$ weeks get CPAP if clinically indicated. If necessary, surfactant can be administered via a thin catheter in the trachea during

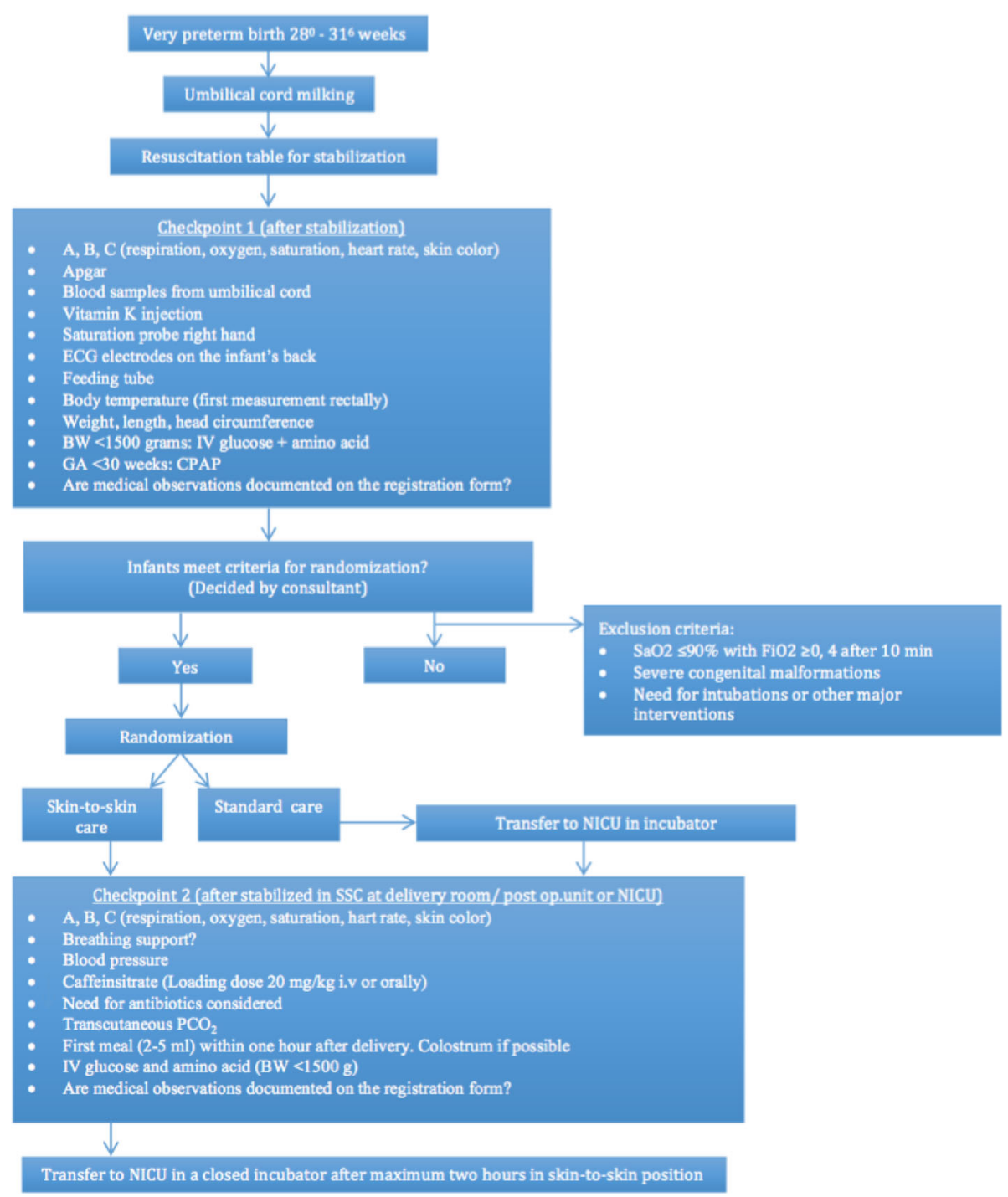

Fig. 1 Flowchart illustrating the first 2 hours after birth 
spontaneous breathing on CPAP ("surfactant without intubation" (SWI) if oxygen requirement exceeds $35 \%$ on $\mathrm{CPAP}$ and/or the infant has signs of moderate to severe respiratory distress. After the infant is stabilized in skin-to-skin position on the mother's chest (SSC) or in a closed incubator and transferred to the NICU (SC), the infant is evaluated again (checkpoint 2) according to the checklist (Fig. 1).

Infants in the intervention group are offered SSC up to 2 hours after delivery - in the delivery room after vaginal delivery, or in the recovery room after C-section. A consultant and a neonatal nurse are responsible for the infant, while a midwife takes care of the mother. After $\mathrm{C}$-section, the same personnel from the NICU and from the maternity ward are present in addition to specialist nurses from the postoperative ward. When the infant is stabilized on the mother's chest, the consultant can leave the DR or postoperative ward in agreement with the neonatal nurse in charge. The consultant is nearby and easily reachable by telephone.

Further treatment during the hospitalization does not differ between groups. According to the unit's guidelines, both mothers and fathers have unrestricted access to the NICU and are encouraged to have as much skin-to-skin contact with their preterm infant as possible.

\section{Simulation-based training (SBT)}

Simulation-based training (SBT) is effective for medical education [31], facilitates multidisciplinary training [32], and can be tailored to individual needs with no risk for human patients. Before study start, simulation-based training was facilitated for five consultants, fifteen neonatal nurses and ten midwives. One gynecologist, one anesthesiologist, and one operating nurse were observers during one SBT. The SBT was a collaboration with the Medical Simulation Center (MSC) at St. Olav's University Hospital/NTNU. The instructors from the MSC (one pediatrician and one specialist nurse) had formal European standard instructor training. An in situ scenario, reflecting the complexity of the admittance of a very preterm infant, was customized and conducted in the OR and DR during a total of seven training days.

Prior to the SBT at St. Olav's Hospital, the teams were informed about the equipment, the environment, and the procedures for the initial stabilization and the intervention. Each trainee participated actively in one or two scenarios per training day. Immediately before the scenario, each team received a case history with information about GA and birth weight, saturation level, heart rate, and the work of breathing. Any change in physiological parameters was visible on a monitor, and the team had to act based on the infant's condition. Each team comprised two neonatal nurses, a consultant in neonatology, and one or two midwives. One person outside the resource team acted as a mother in labor during all training days.

\section{Outcomes measures \\ Primary outcome}

Bayley Scales of Infant and Toddler Development, Third Edition (Bayley-III) The Bayley-III is a screening test that includes assessment of cognition, language (receptive and expressive), and motor function (gross and fine) in infants and young children from 0 to 42 months and provides a measurable and validated cognitive quotient [33]. The occupational therapist conducting the Bayley for ages 1 and 2 years are unaware of the intervention group.

\section{Secondary outcomes}

Safety Hypothermia $\left(<36{ }^{\circ} \mathrm{C}\right)$, respiratory failure requiring acute intubation, and/or cardiopulmonary resuscitation (CPR).

Physiological stability Physiological variables are recorded during the first 24 hours (Table 1).

State-Trait Anxiety Inventory (STAI) Maternal anxiety is measured with STAI Form Y [34]. STAI measures state and trait anxiety [35]; the mothers answer this questionnaire before the infants are discharged from the hospital, and when the child is 3 months and 2 years (corrected age). The questionnaire consists of 20 statements that evaluate how the mother feels 'at this moment' and twenty statements that evaluate how the mother feels 'generally'. All items are scored according to a 4-point Likert scale

General Movement Assessment (GMA) All infants are videotaped at 10-15 weeks post term age (fidgety movements' period) for the GMA and the AMR. The video recordings are performed and classified according to the Prechtl method [36, 37] by a certified GMA observer unaware of the intervention group.

Ages \& Stages Questionnaire - Social-Emotional (ASQ-SE) The child's social and emotional competence is evaluated using the ASQ-SE at corrected age of 3 months and 2 years [38]. The ASQ-SE comprises 22 questions that address seven behavioral areas: self regulation, compliance, communication, adaptive functioning, autonomy, affect, and interaction with people. The ASQ-SE is a screening instrument developed for children aged 3 months to 5 years.

\section{Clinical registration}

A case report form (CRF) has been prepared in cooperation with the Unit for Applied Clinical Research at the 
Norwegian University of Science and Technology (NTNU). The CRF is divided into three parts.

1. On admission and the first 24 hours: Background data which are recorded are: mode of delivery, GA, sex, Apgar scores at 1, 5 and 10 minutes, birthweight, length, head circumference, maternal cause of preterm delivery (preeclampsia, breech position, rupture of membranes, premature contractions or infection), fetal cause of preterm delivery (growth retardation, non-reassuring CTG registration), and antenatal steroids (full or incomplete course).

Observations and interventions which are recorded during the first 24 hours are: oxygen saturation and requirement, mode of breathing support (hourly), surfactant administration, time of SSC after birth and any cause for interrupted SSC before 120 minutes, age at the first feed (gavage or oral), any intravenous infusion, transcutaneous carbon dioxide and total amount of enteral and parenteral nutrition given. Blood pressure is measured once during the first 120 minutes.

Adverse events: the safety is closely monitored by the consultant and the neonatal nurse present. It will be registered as an adverse event if body temperature drops $<36.0 \mathrm{C}^{\circ}$ or if the infants have signs of any respiratory failure requiring interventions in addition to an interruption of SSC and transfer to the NICU for a higher level of monitoring and/or respiratory support.

2. During hospitalization:

Variables recorded are: daily weight, age on removal of feeding tube, time in skin-to-skin position every day, all nutrition (parenteral and enteral), any insulin given, any surgery, mechanical ventilation (mode and duration), CPAP/bilevel positive airway pressure (BiPAP) (duration), supplemental oxygen and/or ventilator support at 28 postnatal days, 36 and 40 weeks postmenstrual age (BPD), any sepsis (with maximal CRP and duration of antibiotic), cerebral ultrasound and magnetic resonance imaging (MRI) results (IVH or PVL), seizures, NEC, treatment for PDA and postmenstrual age (PMA) for transfer from intermediate to the step-down unit in the NICU.

3. At discharge:

Variables recorded are: PMA, weight, length, head circumference and mode and type of nutrition. At St. Olav's Hospital, families living less than 30 minutes by car from the hospital are offered home care with gavage feeding for infants from PMA 34 weeks. Criteria for participation for the infants are: full enteral feeds; no apneas/bradycardias requiring caffeine or monitoring; stable body temperature and positive weight gain. A family is invited to participate in the home care program if there are two caregivers at home during the day and at least one of them speaks Norwegian or English.

\section{Sociodemographic information}

Information about education, employment (part- or fulltime) and marital status is collected from the mothers' medical records.

\section{Consent and enrolment}

Pregnant women admitted to the maternity ward at St. Olav's University Hospital for anticipated preterm delivery between $28^{0}-31^{6}$ weeks of gestation are eligible. Oral and written information about the study is provided by a pediatrician and/or a neonatal nurse, and written consent is obtained before delivery,

\section{Randomization and allocation concealment}

The randomization is done after the initial stabilization of the infant (Fig. 1). Infants are stratified by weeks of gestation $\left(28^{0}-29^{6}\right.$ and $\left.30^{0}-31^{6}\right)$. The randomization is conducted using sealed envelopes organized by the Unit for Applied Clinical Research at the NTNU.

\section{Sample size and statistical analyses}

Primary analysis: sample size calculations are performed for a two-sample $t$ test for comparing the Bayley Scale of Infant and Toddler Development, Third Edition, cognitive scale between the intervention (SSC) and control (SC) groups at 2 years of corrected age. To obtain a power of $80 \%$ for detecting a difference of 7.5 in mean score, using SD $=15$ and significance level $\alpha=0.05,64$ preterm infants are needed in each group. To allow for withdrawals, the sample size is set to 68 in each group.

Secondary analysis: differences between the SSC and SC groups for continuous variables will be analyzed using two-sample $t$ tests, or Mann-Whitney $U$ tests for data with non-normal distributions. Categorical variables will be analyzed by the Pearson chi-square test or Fisher's exact test. $P$ values $<0.05$ are considered statistically significant. Due consideration of multiple testing will be made when interpreting the results. All data will be presented and analyzed in accordance with the updated CONSORT guidelines for randomized trials [39].

\section{Discussion}

The presentation of the study protocol covering design, outcome measures, sample size calculations, and procedures of this RCT on early skin-to-skin for very preterm infants, is in accordance with the Standard Protocol Items: Recommendations For Interventional Trials (SPIRIT) 2013 statement for clinical trial protocols Additional file 1 [40]. 
Bayley Scales of Infant and Toddler Development, Third Edition (Bayley-III) at 2 years of corrected age is chosen as the primary outcome measure. Although many interventions in the neonatal period appear to have short-term beneficial effects [15, 23, 41, 42], such effects seem to disappear over time [15, 43]. The complexity of neurodevelopment and the genetic, epigenetic, and environmental factors, which may influence the development, makes it less likely that a single, short-lasting intervention will lead to a change in cognition and/or behavior after 2 years. Thus, any intervention offered to these infants must be followed over time to learn more about their potential effects or lack of such.

In order to assess developmental delay in the children at the corrected age of 3 months and 2 years, the ASQ$\mathrm{SE}$ is used [38]. This screening instrument is found to be valid in identifying of social and emotional difficulties in children [44]. Preterm delivery is associated with maternal anxiety and stress [45-47], and the effect of separation of the mother and her very preterm infant is not well studied. We have chosen to assess this aspect by using the STAI, which is validated in a Norwegian population and found reliable in terms of assessing anxiety in women [34].

Lately, there has been a shift toward a more gentle initial handling of preterm neonates. This view is well described by Jobe and colleagues, who argue that the majority of preterm infants need only supportive treatment in the transition to the extrauterine life, and not resuscitation $[48,49]$. The main focus of this approach has, so far, been on early CPAP $[50,51]$, less invasive ways of surfactant administration [52, 53], avoidance of early mechanical ventilation [54], and maintenance of adequate body temperature [55]. In line with this, facilitation of early skin-to-skin contact between the preterm infant and the mother could contribute to a more gentle adjustment to extrauterine life with improved physiological stability. The results from this study will have important implications for how we care for very preterm infants immediately after delivery. It will also increase our understanding of how early SSC affects very preterm infants and their mothers.

Those who are conducting the Bayley-III and the GMA will be masked to the intervention group, but not the parents and NICU staff caring for the baby in the first 24 hours. Parents in the SSG group may continue to have an increased focus on SSC, and this could potentially extend the total skin-to-skin time in the NICU. However, this limitation seems inevitable with the intervention in question.

\section{Trial status}

The study is currently recruiting participants. Since study start February 1, 2014 and through June 2016, 67 eligible pregnant women have been admitted to the maternity ward at St. Olav's Hospital. A majority of these did not deliver before 32 weeks of gestation. Up to June, 2016, 27 infants have been included in the study.

From January 1, 2017, two other hospitals in Norway will participate in the study: Drammen Hospital, located in the eastern part of Norway, and Kristiansand Hospital in the southern part. Between 30 and 35 infants with GA $<32$ weeks are born every year at each of these hospitals. Based on this, the estimated time for end of inclusion will be 2018 .

\section{Additional file}

Additional file 1: SPIRIT 2013 Checklist: recommended items to address in a clinical trial protocol and related documents*. (PDF $74 \mathrm{~kb}$ )

\section{Abbreviations}

AMR: Assessment of Motor Repertoire; ASQ-SE: Ages \& Stages Questionnaire Social-Emotional; Bayley-III: Bayley Scales of Infant and Toddler Development, Third Edition; BPD: Bronchopulmonary dysplasia; CPAP: Continuous positive airway pressure; CRF: Case report form; CRP: C-reactive protein; DR: Delivery room; FFC: Family-centered care; GA: Gestational age; GMA: General Movement Assessment; IVH: Intraventricular hemorrhage; KC: Kangaroo care; NEC: Necrotizing enterocolitis; NICU: Neonatal intensive care unit; NIDCAP: Newborn Individualized Developmental Assessment Program; OR: Operation room; PDA: Persistent ductus arteriosus; PMA: Postmenstrual age; PVL: Periventricular leukomalacia; SBT: Simulation-based training; SC: Standard care; SSC: Skin-to-skin care; STAl: State-Trait Anxiety Inventory

\section{Acknowledgements}

Not applicable.

Funding

Not applicable. The study does not receive any financial support.

Availability of data and materials

Not applicable.

\section{Authors' contributions}

LK is project leader of the study, and contributed significantly to the study design, data management and collection, ethics considerations, and implementation strategies, and drafted the manuscript. HB significantly contributed to the study design, development of the intervention, outcomes, sample size calculation, data collection and management, and made substantive intellectual contribution in writing, and supervision. RS significantly contributed to the study design, development of the intervention, outcomes, sample size calculation, data collection and management and made substantive intellectual contribution to writing the manuscript. TF contributed significantly to sample size calculation, statistical analysis proposal, and randomization processes. MS helped to develop the concept, study design, and outcomes. HR was involved in the study design, data collection, and management. HSM was involved in implementation strategies and study design. IN was involved in development of the feasibility objectives, and study design. All authors were involved in the review of this manuscript and have seen and are in agreement with the final manuscript.

\section{Competing interests}

The authors declare that they have no competing interests.

\section{Consent for publication}

Written informed consent was obtained from the patient/participant for publication of their individual details and accompanying images in this manuscript. The consent form is held by the authors, and is available for review by the Editor-in-Chief. 


\section{Ethics approval and consent to participate}

This study has been performed in accordance with the Declaration of Helsinki and has been approved by the Regional Committee of Ethics in Medical Research (Mid-Norway) (2013/638/REK midt).

\section{Author details}

'Department of Pediatrics, St. Olav's University Hospital, Trondheim, Norway. 2Department of Laboratory Medicine, Children's and Women's Health, Norwegian University of Science and Technology, NTNU, Trondheim, Norway. ${ }^{3}$ Department of Clinical Services, St. Olav's University Hospital, Trondheim, Norway. ${ }^{4}$ Department of Public Health and General Practice, NTNU, Trondheim, Norway. ${ }^{5}$ Department of Circulation and Medical Imaging, Norwegian University of Science and Technology, NTNU, Trondheim, Norway. ${ }^{6}$ Department of Anesthesia and Intensive Care Medicine, St. Olav's University Hospital, Trondheim, Norway.

\section{Received: 8 July 2016 Accepted: 24 November 2016} Published online: 12 December 2016

\section{References}

1. Blencowe H, Cousens $\mathrm{S}$, Oestergaard MZ, Chou D, Moller AB, Narwal R, et al. National, regional, and worldwide estimates of preterm birth rates in the year 2010 with time trends since 1990 for selected countries: a systematic analysis and implications. Lancet. 2012;379(9832):2162-72.

2. Lawn JE, Wilczynska-Ketende K, Cousens SN. Estimating the causes of 4 million neonatal deaths in the year 2000. Int J Epidemiol. 2006:35(3):706-18.

3. Goldenberg RL, Culhane JF, lams JD, Romero R. Epidemiology and causes of preterm birth. Lancet. 2008;371(9606):75-84.

4. Mwaniki MK, Atieno M, Lawn JE, Newton CR. Long-term neurodevelopmenta outcomes after intrauterine and neonatal insults: a systematic review. Lancet 2012;379(9814):445-52

5. Spittle A, Orton J, Anderson P, Boyd R, Doyle LW. Early developmental intervention programmes post-hospital discharge to prevent motor and cognitive impairments in preterm infants. Cochrane Database Syst Rev. 2012;12:CD005495.

6. Lund LK, Vik T, Lydersen S, Lohaugen GC, Skranes J, Brubakk AM, et al. Mental health, quality of life and social relations in young adults born with low birth weight. Health Qual Life Outcomes. 2012;10:146.

7. Dahl LB, Kaaresen PI, Tunby J, Handegard BH, Kvernmo S, Ronning JA. Emotional, behavioral, social, and academic outcomes in adolescents born with very low birth weight. Pediatrics. 2006;118(2):e449-59.

8. Hack M. Young adult outcomes of very-low-birth-weight children. Semin Fetal Neonatal Med. 2006;11(2):127-37.

9. Hack M, Youngstrom EA, Cartar L, Schluchter M, Taylor HG, Flannery D, et al. Behavioral outcomes and evidence of psychopathology among very low birth weight infants at age 20 years. Pediatrics. 2004;114(4):932-40.

10. Husby IM, Skranes J, Olsen A, Brubakk AM, Evensen KA. Motor skills at 23 years of age in young adults born preterm with very low birth weight. Early Hum Dev. 2013;89(9):747-54.

11. Saigal S, Doyle LW. An overview of mortality and sequelae of preterm birth from infancy to adulthood. Lancet. 2008;371(9608):261-9.

12. Evensen KA, Vik T, Helbostad J, Indredavik MS, Kulseng S, Brubakk AM. Motor skills in adolescents with low birth weight. Arch Dis Child Fetal Neonatal Ed. 2004;89(5):F451-5.

13. Hack M, Flannery DJ, Schluchter M, Cartar L, Borawski E, Klein N. Outcomes in young adulthood for very-low-birth-weight infants. N Engl J Med. 2002; 346(3):149-57.

14. Berlin LJ, Brooks-Gunn J, McCarton C, McCormick MC. The effectiveness of early intervention: examining risk factors and pathways to enhanced development. Prev Med. 1998;27(2):238-45.

15. Vanderveen JA, Bassler D, Robertson CM, Kirpalani H. Early interventions involving parents to improve neurodevelopmental outcomes of premature infants: a meta-analysis. J Perinatol. 2009;29(5):343-51.

16. Mehler K, Wendrich D, Kissgen R, Roth B, Oberthuer A, Pillekamp F, et al. Mothers seeing their VLBW infants within $3 \mathrm{~h}$ after birth are more likely to establish a secure attachment behavior: evidence of a sensitive period with preterm infants? J Perinatol. 2011;31(6):404-10.

17. Moore KA, Coker K, DuBuisson AB, Swett B, Edwards WH. Implementing potentially better practices for improving family-centered care in neonatal intensive care units: successes and challenges. Pediatrics. 2003;111(4 Pt 2): e450-60.
18. Nuss T, Kelly KM, Campbell KR, Pierce C, Entzminger JK, Blair BK, et al. The impact of opening visitation access on patient and family experience. J Nurs Adm. 2014;44(7/8):403-10.

19. Nyqvist KH, Anderson GC, Bergman N, Cattaneo A, Charpak N, Davanzo R, et al. Towards universal Kangaroo Mother Care: recommendations and report from the First European conference and Seventh International Workshop on Kangaroo Mother Care. Acta Paediatr. 2010;99(6):820-6.

20. Harrison $\mathrm{H}$. The principles for family-centered neonatal care. Pediatrics. 1993;92(5):643-50.

21. Als H. Toward a synactive theory of development: promise for the assessment of infant individuality. Infant Ment Health J. 1982;3:229-43.

22. Als H. A synactive model of neonatal behavioral organization: framework for the assessment of neurobehavioral development in the premature infant and for support of infants and parents in the neonatal intensive care environment. Phys Occup Ther Pediatr. 1986:6(3-4):3-53.

23. Nordhov SM, Ronning JA, Dahl LB, Ulvund SE, Tunby J, Kaaresen PI. Early intervention improves cognitive outcomes for preterm infants: randomized controlled trial. Pediatrics. 2010;126(5):e1088-94.

24. Nordhov SM, Ronning JA, Ulvund SE, Dahl LB, Kaaresen PI. Early intervention improves behavioral outcomes for preterm infants: randomized controlled trial. Pediatrics. 2012;129(1):e9-16

25. World Health Organization/UNICEF. Baby-friendly hospital initiative: revised, updated and expanded for integrated care. 2009 Available from: http:// www.who.int/nutrition/publications/infantfeeding/bfhi_trainingcourse/en/. Accessed 18 Sept 2015.

26. Bergman NJ, Linley LL, Fawcus SR. Randomized controlled trial of skin-to-skin contact from birth versus conventional incubator for physiological stabilization in 1200- to 2199-gram newborns. Acta Paediatr. 2004;93(6):779-85.

27. Chi Luong K, Long Nguyen T, Huynh Thi DH, Carrara HP, Bergman NJ. Newly born low birthweight infants stabilise better in skin-to-skin contact than when separated from their mothers: a randomised controlled trial. Acta Paediatr. 2016;105(4):381-90.

28. Morelius E, Ortenstrand A, Theodorsson E, Frostell A. A randomised trial of continuous skin-to-skin contact after preterm birth and the effects on salivary cortisol, parental stress, depression, and breastfeeding. Early Hum Dev. 2015;91(1):63-70

29. World Health Organization. WHO recommendations on interventions to improve preterm birth outcomes. Geneva: World Health Organization; 2015 [Available from: http://apps.who.int/iris/bitstream/10665/183037/1/ 9789241508988_eng.pdf?ua=1. Accessed 15 Dec 2015.

30. Kristoffersen L, Stoen R, Hansen LF, Wilhelmsen J, Bergseng H. Skin-to-skin care after birth for moderately preterm infants. J Obstet Gynecol Neonatal Nurs. 2016;45(3):339-45.

31. Cook DA, Hatala R, Brydges R, Zendejas B, Szostek JH, Wang AT, et al. Technology-enhanced simulation for health professions education: a systematic review and meta-analysis. JAMA. 2011;306(9):978-88.

32. Halamek LP. The simulated delivery-room environment as the future modality for acquiring and maintaining skills in fetal and neonatal resuscitation. Semin Fetal Neonatal Med. 2008;13(6):448-53.

33. Bayley N. Bayley Scales of Infant and Toddler Development. San Antonio: Harcourt Assessment; 2005.

34. Håseth K, Hagtvedt K, Spielberger C. Manual for Norwegian State-Trait Anxiety Inventory. Unpublished document. Psychological Institute University of Oslo. 1993.

35. Spielberger CD, Gorsuch RL, Lushene R, Vagg PR, Jacobs GA. Manual for the State-Trait Anxiety Inventory. Palo Alto: Consulting Psychologists; 1983.

36. Einspieler C, Prechtl HF, Bos AF, Ferrari F, Cioni G. Prechtl's method on the qualitative assessment of general movements in preterm, term and young infants. New York: Mac Keith; 2004.

37. Ferrari F, Cioni G, Prechtl HF. Qualitative changes of general movements in preterm infants with brain lesions. Early Hum Dev. 1990;23(3):193-231.

38. Squires J, Bricker D, Heo K, Twombly E. Ages \& Stages Questionnaires: Social-Emotional (ASQ:SE). A parent-completed, child-monitoring system for social-emotional behaviors. Baltimore: Brookes; 2002.

39. Schulz KF, Altman DG, Moher D. CONSORT 2010 statement: updated guidelines for reporting parallel group randomised trials. Int J Surg. 2011;9(8):672-7.

40. Agha RA, Altman DG, Rosin D. The SPIRIT 2013 statement-defining standard protocol items for trials. Int J Surg. 2015;13:288-91.

41. Bonnier C. Evaluation of early stimulation programs for enhancing brain development. Acta Paediatr. 2008;97(7):853-8. 
42. Boundy EO, Dastjerdi R, Spiegelman D, Fawzi WW, Missmer SA, Lieberman E, et al. Kangaroo mother care and neonatal outcomes: a meta-analysis. Pediatrics. 2016;137(1):1-16.

43. Hauglann L, Handegaard BH, Ulvund SE, Nordhov M, Ronning JA, Kaaresen PI. Cognitive outcome of early intervention in preterms at 7 and 9 years of age: a randomised controlled trial. Arch Dis Child Fetal Neonatal Ed. 2015; 100(1):F11-6.

44. Briggs RD, Stettler EM, Silver EJ, Schrag RD, Nayak M, Chinitz S, et al. Socialemotional screening for infants and toddlers in primary care. Pediatrics. 2012;129(2):e377-84.

45. Kaaresen PI, Ronning JA, Ulvund SE, Dahl LB. A randomized, controlled trial of the effectiveness of an early-intervention program in reducing parenting stress after preterm birth. Pediatrics. 2006;118(1):e9-19.

46. Misund AR, Nerdrum P, Diseth TH. Mental health in women experiencing preterm birth. BMC Pregnancy Childbirth. 2014;14:263.

47. Spear ML, Leef K, Epps S, Locke R. Family reactions during infants' hospitalization in the neonatal intensive care unit. Am J Perinatol. 2002;19(4):205-13.

48. Jobe AH. Transition/adaptation in the delivery room and less RDS: "Don't just do something, stand there!". J Pediatr. 2005;147(3):284-6.

49. O'Donnell CP. 'Resuscitation' of extremely preterm and/or low-birth-weight infants - time to 'call it'? Neonatology. 2008;93(4):295-301.

50. DeMauro SB, Douglas E, Karp K, Schmidt B, Patel J, Kronberger A, et al. Improving delivery room management for very preterm infants. Pediatrics. 2013;132(4):e1018-25.

51. Morley CJ, Davis PG, Doyle LW, Brion LP, Hascoet JM, Carlin JB. Nasal CPAP or intubation at birth for very preterm infants. N Engl J Med. 2008:358(7):700-8.

52. Cools F. A new method of surfactant administration in preterm infants. Lancet. 2011;378(9803):1607-8.

53. Gopel W, Kribs A, Hartel C, Avenarius S, Teig N, Groneck P, et al. Less invasive surfactant administration is associated with improved pulmonary outcomes in spontaneously breathing preterm infants. Acta Paediatr. 2015; 104(3):241-6.

54. Gopel W, Kribs A, Ziegler A, Laux R, Hoehn T, Wieg C, et al. Avoidance of mechanical ventilation by surfactant treatment of spontaneously breathing preterm infants (AMV): an open-label, randomised, controlled trial. Lancet. 2011;378(9803):1627-34.

55. Vento M, Lista G. Managing preterm infants in the first minutes of life. Paediatr Respir Rev. 2015;16(3):151-6.

\section{Submit your next manuscript to BioMed Central and we will help you at every step:}

- We accept pre-submission inquiries

- Our selector tool helps you to find the most relevant journal

- We provide round the clock customer support

- Convenient online submission

- Thorough peer review

- Inclusion in PubMed and all major indexing services

- Maximum visibility for your research

Submit your manuscript at www.biomedcentral.com/submit 